\title{
Author Index to Volume 5
}

Boon A 27

Chan V 47

Chih-Feng Shu S 177

de Holanda F 189

Desyllas J 61

Frey HW 13

Ganesan S 3
Gerhardt W 233

Greene M 61

Hall AC 123

Hanson J 97

Heitor T 199

Heng CK 47

Hillier B 61

Jenks M 233
Karimi K 221

Kruger M 199

Lau SSY 3

Muchagato J 199

Ramos T 199

Read S 209

Reeve A 141
Rigatti D 161

Simmonds R 141

Temple M 27

Tostes A 199

Zhang XQ 37 